\title{
Busulfan Dosing Literature Review
}

Cynthia L Gong ${ }^{1 *}$, Rezvani $A R^{1,2}, \operatorname{Kim~T}^{1}$ and Studdert $\mathrm{AL}^{3}$

${ }^{1}$ Department of Pharmacy, Stanford Health Care, Stanford, California, USA

${ }^{2}$ Stanford University School of Medicine, Stanford, California, USA

${ }^{3}$ Stanford Health Care, Center for High Value Care, Stanford, California, USA

\begin{abstract}
Introduction: There is debate regarding the use of busulfan every 6 hours ( $q 6 h$ ) compared to every 24 hours (q24 $\mathrm{h}$ ) in patients undergoing hematopoietic cell transplantation.

Objectives: To review the literature to determine whether there is a significant difference between dosing busulfan q6 $\mathrm{h}$ vs. q24 $\mathrm{h}$ in adults, and to review dosing strategies to optimize daily dosing.

Methods: A literature search was conducted in PubMed with the terms "busulfan" and "transplant" and "24" in all fields. Results were further refined by using the terms "busulfan" and "transplant" and "pharmacokinetics". Titles were then reviewed for relevance, and the remaining articles reviewed by abstract. Articles deemed relevant were then read in more thorough detail, and references cited by these articles reviewed to ensure a comprehensive review of the literature. Studies focusing on the pediatric population were not reviewed.

Results: 478 articles were identified, and of these, 372 contained the term "pharmacokinetics". Based on abstract review, 26 relevant articles were identified. All articles confirmed that there are no differences in the pharmacokinetics of q6 h vs. q24 h dosing, and that safety appears equivalent between the two dosing schemes. One study noted an increase in the occurrence of acute graft-vs.-host disease (GVHD) and possibly increased gastrointestinal $(\mathrm{Gl})$ toxicity and stomatitis with $\mathrm{q} 6 \mathrm{~h}$ dosing, while another noted a higher incidence of toxicity with q24 $\mathrm{h}$ dosing specifically in metastatic renal cell carcinoma patients. All articles concluded that both regimens are equally effective, but that q24 h dosing is more convenient and likely to decrease hospitalization, nursing, and pharmacy requirements.
\end{abstract}

Conclusions: There is no difference in efficacy or safety between busulfan q6 $\mathrm{h}$ and $\mathrm{q} 24 \mathrm{~h}$ dosing. Institutions should consider moving to daily dosing of busulfan for improved convenience and decreased costs.

Keywords: Busulfan; Pharmocokinetics; Myelogenous leukemia

\section{Introduction}

Busulfan utilization has undergone dramatic progress in hematopoietic cell transplant (HCT) since its initial approval in 1954 [1]. Busulfan is an alkylating agent originally used in chronic myelogenous leukemia (CML), but it has progressively been recognized as a potent myeloablative agent in preparative regimens for hematopoietic cell transplantation (HCT) $[2,3]$. Busulfan-containing regimens have been widely accepted as a standard of care, and represent the most frequently used myeloablative regimens prior to HCT $[4,5]$

Busulfan tablets on the market are available only in much smaller doses than those necessary for HCT conditioning [6], as the oral busulfan formulation was originally intended for the CML population [7-9]. This results in a high pill burden for patients on HCT conditioning regimens. Concerns regarding adherence, requiring patients to consume large quantities of pills in a single setting, and the variable systemic bioavailability resulting from a large single oral dose of busulfan led to the development of conditioning protocols that utilize multiple oral doses spread throughout the day, typically at 6-hour intervals [10-13].

Eventually, as high-dose busulfan emerged as an important component of preparative regimens in the early 2000s, intravenous formulations were marketed to overcome the disadvantages of the original oral compound's bioavailability [14]. Alkylating agents are typically dosed at a daily frequency due to cell cycle non-specific activity, and complete bioavailability and elimination of pill burden could theoretically eliminate the necessity of multiple doses in a day [15-25]. Nonetheless, studies comparing intravenous versus oral administration of busulfan used identical dosing frequencies (again, typically $\mathrm{q} 6 \mathrm{~h}$ ) in their protocols to avoid confounding variables [26]. Further, it has been found that IV busulfan produces more reliable pharmacokinetic parameters than oral dosing due to its immediate bioavailability and independence from administration with food [27].

There are many disadvantages to high-frequency busulfan dosing. Patients are disturbed more frequently with overnight chemotherapy administrations, possibly impacting recovery and satisfaction. Institutions carry a heavier burden with the resources necessary for admixture, delivery, and nursing administration times with multiple doses per day versus once-daily dosing. Waste can be a significant cost to institutions, since drug waste is produced with each admixture [28]. Due to busulfan's relative short stability, frequent administrations are difficult to batch together [29]. Logically, decreasing the frequency of administration has the potential to improve patient satisfaction, improve convenience, and reduce waste (Figure 1).

As busulfan has been incorporated into more conditioning regimens and additional pharmacokinetic data becomes available,

*Corresponding author: Cynthia L Gong, Department of Pharmacy, Stanford Health Care, 300 Pasteur Drive, MC5275, Stanford, California, USA, Tel: (650) 206-3563/(650) 736-1749; Fax: (650) 724-8674; E-mail: cgong@stanfordhealthcare.org

Received January 19, 2018; Accepted February 05, 2018; Published February 12, 2018

Citation: Gong CL, Rezvani AR, Kim T, Studdert AL (2018) Busulfan Dosing Literature Review. J Blood Lymph 8: 204. doi:10.4172/2165-7831.1000204

Copyright: ( 2018 Gong CL, et al. This is an open-access article distributed unde the terms of the Creative Commons Attribution License, which permits unrestricted use, distribution, and reproduction in any medium, provided the original author and source are credited. 
the literature has become more robust with evidence supporting once daily busulfan dosing [30]. To address concerns regarding the safety and efficacy of busulfan IV every six hours ( $\mathrm{q} 6 \mathrm{~h}$ ) compared to every 24 hours (q24 h), we undertook a literature review comparing the two dosing regimens. The objective was to assess the safety, efficacy, and pharmacokinetics to warrant a conversion of regimens that dose busulfan $\mathrm{q} 6 \mathrm{~h}$ to an equivalent once-daily intravenous dose.

\section{Methods}

Using PubMed, a literature search was conducted with the terms "busulfan" and "transplant" and " 24 " in all fields. Results were further refined by using the terms "busulfan" and "transplant" and "pharmacokinetics". Titles were then reviewed for relevance, and the remaining articles reviewed by abstract. Articles deemed relevant were then read thoroughly, and any additional references cited by these articles were reviewed to ensure a comprehensive review of the literature. Figure 1 depicts the search strategy used for this review.

\section{Results}

478 articles were identified in the initial search using "busulfan" and "transplant" and " 24 ". When the terms were changed to include "pharmacokinetics", 372 articles were identified. Review of abstracts and filter by English language yielded 26 articles relevant to the comparison of IV busulfan $\mathrm{q} 6 \mathrm{~h}$ vs. q $24 \mathrm{~h}$, or discussed optimal dosing strategies using q24 h dosing. All articles except one concluded that q24 $\mathrm{h}$ dosing was feasible, safe, and convenient for both adults and pediatric patients, with clinical equivalence to $\mathrm{q} 6 \mathrm{~h}$ dosing. Of the studies reviewed, two found higher rates of toxicity in the $\mathrm{q} 24 \mathrm{~h}$ compared to the $\mathrm{q} 6 \mathrm{~h}$ regimen $[31,32]$.

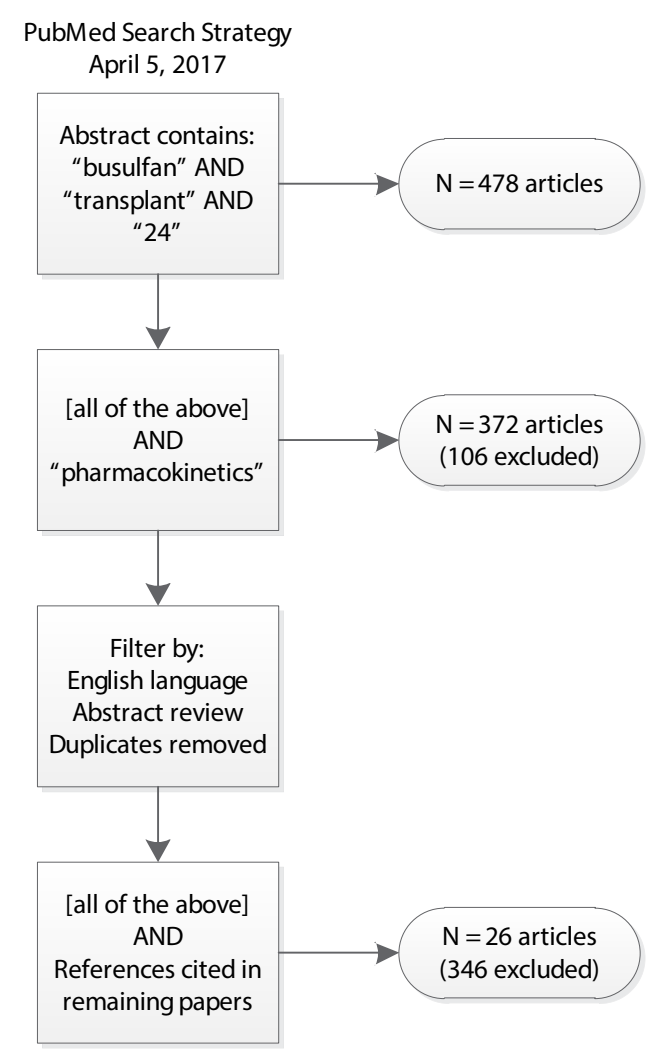

Figure 1: Search Strategy using PubMed.
One study that found that $\mathrm{q} 24 \mathrm{~h}$ dosing had unacceptable toxicity was done in a small sample of seven metastatic renal cell carcinoma patients, six of whom had undergone previous unilateral nephrectomy, and one of whom had renal embolization of a diseased kidney at the time of diagnosis [32]. The authors acknowledged that a combination of kidney deficiency combined with previous exposure to interleukin-2 and combination busulfan with fludarabine likely contributed to the toxicities experienced in their study.

The second study was done in pediatrics, and found that the rate of veno-occlusive disease (VOD) was higher in the q24 h group compared to the $\mathrm{q} 6 \mathrm{~h}$ group [31]. However, the same study found that $\mathrm{q} 24 \mathrm{~h}$ dosing was a predictor for higher event-free survival and overall survival, and recommended that busulfan IV with therapeutic dose monitoring be used over oral busulfan in children undergoing allogeneic stem cell transplantation, particularly among those at high risk for graft failure or relapse [31].

While an additional study found that the incidence of GVHD tended to be slightly higher in patients receiving busulfan IV q24 h, the authors attributed this to the fact that patients in the $\mathrm{q} 6 \mathrm{~h}$ group were younger, and that there was a higher percentage of HLA-matched donors in the $\mathrm{q} 6 \mathrm{~h}$ group. This difference was not statistically significant [5].

Overall, most studies focused on the adult population, primarily with AML or CML. Only 3 studies looked at pediatrics, with a collective $\mathrm{n}=109$ patients $[31,33,34]$. Most studies were done to evaluate the pharmacokinetics of a once-daily dosing regimen. Of these studies, those focusing on pharmacokinetics found that there were either no significant differences in overall pharmacokinetic parameters, or that there was no difference in target area-under-the-curve (AUC) between the two dosing regimens. Although some minor differences were found among the studies for the initial administered dose, most studies evaluated an empiric weight-based dosing regimen of $3.2 \mathrm{mg} / \mathrm{kg} \mathrm{IV} \mathrm{q24}$ h. Only four studies evaluating busulfan in adults used a dose based on body surface area [35-38]. Despite these differences, pharmacokinetic analysis remains a mandatory component of busulfan administration in order to reach the appropriate target AUC and reduce inter-individual variability [31].

A summary of relevant literature is shown in Table 1.

\section{Discussion}

IV busulfan has been a standard myeloablative regimen for HCT for many years. Based on our literature review, dosing busulfan q24 h is comparable in therapeutic efficacy and pharmacokinetic profile to busulfan $\mathrm{q} 6 \mathrm{~h}$.

In in-vitro pharmacodynamic experiments, it appears that the cell's response to busulfan depends on both concentration and time of exposure [39]. As neither of these factors is exclusive of one another, AUC exposure seems to represent a more relevant pharmacokinetic criterion to correlate its cellular effects. Furthermore, in those same studies, cellular death seemed to not be affected by the dosing schema used to achieve the overall AUC [39].

Anecdotally, we have observed increased nausea and vomiting associated with increased antiemetic utilization, indicating that maximum drug concentration may have a more significant adverse impact on certain organ systems, rather than time of exposure for those systems. Current reports evaluating the toxicity profile between the two dosing regimens focus on major events such as neurotoxicity, 


\begin{tabular}{|c|c|c|c|c|}
\hline Population & Busulfan Dosing Schedule & $\begin{array}{l}\text { Pharmacokinetic } \\
\text { Parameters }\end{array}$ & Safety & Conclusion \\
\hline $\begin{array}{l}\text { Adults with } \mathrm{NHL}, \mathrm{AML} \text {, } \\
\text { or } \mathrm{CML}\end{array}$ & $\begin{array}{l}\text { 1. Bu } 1.6 \mathrm{mg} / \mathrm{kg} \text { IV q12 h × } 4 \text { days }(\mathrm{n}=6) \\
\text { 2. Bu } 3.2 \mathrm{mg} / \mathrm{kg} \text { IV q24 h × } 4 \text { days }(\mathrm{n}=6) \\
\text { 3. } 3 \text {. All received Cy } 60 \mathrm{mg} / \mathrm{kg} \text { IV q } 24 \mathrm{~h} \\
\times 2 \text { days }\end{array}$ & $\begin{array}{l}\text { Clearance, half-life, } C_{\text {max' }} \\
\text { and AUC were highly } \\
\text { predictive of later dose PK } \\
\text { profiles }\end{array}$ & $\begin{array}{l}\text { No CNS or pulmonary } \\
\text { toxicity noted }\end{array}$ & $\begin{array}{l}\text { Bu IV can be given safely with } \\
\text { reproducible results on a twice-daily } \\
\text { divided or single-daily dosing schedule to } \\
\text { patients undergoing HSCT. }\end{array}$ \\
\hline $\begin{array}{l}\text { Adults with } A M L, C M L \\
\text { MDS, or CLL }\end{array}$ & $\begin{array}{l}\mathrm{Bu} 3.2 \mathrm{mg} / \mathrm{kg} \text { IV q24 } \mathrm{h} \times 4 \text { days+Flu } 50 \\
\mathrm{mg} / \mathrm{m}^{2} \mathrm{IV} \text { q24 h } \times 4 \text { days }(\mathrm{n}=70)\end{array}$ & $\begin{array}{l}\text { The cumulative AUC was } \\
\text { comparable to the target } \\
\text { range established for } \mathrm{Bu} \\
\mathrm{PO}\end{array}$ & $\begin{array}{l}\text { No unexpected or unusual } \\
\text { toxicity noted }\end{array}$ & $\begin{array}{l}\text { Bu IV is convenient to give, is relatively } \\
\text { well tolerated, and gives predictable blood } \\
\text { levels }\end{array}$ \\
\hline Adults with AML or MDS & $\begin{array}{l}\text { Bu } 130 \mathrm{mg} / \mathrm{m}^{2} \mathrm{IV} \text { q24 } \mathrm{h} \times 4 \text { days+Flu } \\
40 \mathrm{mg} / \mathrm{m}^{2} \text { IV q24 } \mathrm{h} \times 4 \text { days }(\mathrm{n}=96)\end{array}$ & $\begin{array}{l}\text { Clearance in }<24 \text { hours } \\
\text { with no accumulation and } \\
\text { little interdose variation in } \\
\text { PK parameters }\end{array}$ & $\begin{array}{l}\text { Well-tolerated, with only one } \\
\text { death due to regimen-related } \\
\text { complications }\end{array}$ & $\begin{array}{l}\text { Bu IV q24 h yields reproducible and } \\
\text { predictable PK with less interdose/ } \\
\text { interpatient variation vs. Bu PO, and is } \\
\text { efficacious with reduced toxicity. }\end{array}$ \\
\hline $\begin{array}{l}\text { Adults with } \\
\text { hematological } \\
\text { malignancies }\end{array}$ & $\begin{array}{l}\mathrm{Bu} 3.2 \mathrm{mg} / \mathrm{kg} \text { IV q24 } \mathrm{h} \times 4 \text { days }+ \text { Flu } 30 \\
\mathrm{mg} / \mathrm{m}^{2} \mathrm{IV} \times 4 \text { days }(\mathrm{n}=10)\end{array}$ & $\begin{array}{l}\mathrm{C}_{\text {ss }} \text { target and AUC were } \\
\text { comparable to previous } \\
\text { studies using fludarabine } \\
\text { and oral busulfan }\end{array}$ & $\begin{array}{l}\text { One case of primary graft } \\
\text { failure, and one case of } \\
\text { Grade } 4 \text { hyperbilirubinemia }\end{array}$ & $\begin{array}{l}\text { Targeting single-dose Bu IV yields lower } \\
\text { interpatient variability. }\end{array}$ \\
\hline $\begin{array}{l}\text { Adults with acute } \\
\text { leukemias, CML, NHL, } \\
\text { MM, MDS, systemic } \\
\text { mastocytosis, or } \\
\text { myelofibrosis }\end{array}$ & $\begin{array}{l}\text { 1. Bu } 3.2 \mathrm{mg} / \mathrm{kg} \text { IV q24 } \mathrm{h} \times 4 \text { days } \\
(\mathrm{n}=20) \\
\text { 2. Bu } 0.8 \mathrm{mg} / \mathrm{kg} \text { IV q6 } \mathrm{h} \times 4 \text { days }(\mathrm{n}=11) \\
\text { 3. Bu } 1 \mathrm{mg} / \mathrm{kg} \text { PO } \mathrm{q} 6 \mathrm{~h} \times 4 \text { days }(\mathrm{n}=25) \\
\text { 4. All received Cy } 60 \mathrm{mg} / \mathrm{kg} \text { IV q24 } \mathrm{h} \\
\times 2 \text { days }\end{array}$ & $\begin{array}{l}\text { Bu IV q24 } \mathrm{h} \text { is clinically } \\
\text { equivalent to q6 } \mathrm{h} \text { dosing, } \\
\text { with predictable PK } \\
\text { parameters specifically } \\
\text { for those with actual body } \\
\text { weight } \leq 20 \% \text { IBW. }\end{array}$ & $\begin{array}{l}\text { Bu IV q24 h had the least } \\
\text { amount of GVHD. There } \\
\text { were no differences in the } \\
\text { incidence of neurologic } \\
\text { toxicity, hepatic toxicity, } \\
\text { hematologic engraftment, } \\
\text { and relapse at } 100 \text { days. }\end{array}$ & $\begin{array}{l}\text { Bu IV q24 h is safe, convenient, and } \\
\text { consistent for outpatient administration. }\end{array}$ \\
\hline $\begin{array}{l}\text { Pediatrics with } \\
\text { malignant and non- } \\
\text { malignant conditions }\end{array}$ & $\begin{array}{l}\text { 1. Bu } 3.2 \mathrm{mg} / \mathrm{kg} \mathrm{IV} \mathrm{q} 24 \mathrm{~h} \times 2 \text { days }+\mathrm{Flu} \\
30 \mathrm{mg} / \mathrm{m}^{2} \mathrm{IV} \times 5 \text { days }(\mathrm{n}=30) \\
\text { 2. All received a test dose of Bu } 0.8 \mathrm{mg} / \\
\mathrm{kg} \mathrm{IV} \times 1\end{array}$ & $\begin{array}{l}\text { Target AUC and clearance } \\
\text { were achieved using } \\
\text { information from a test } \\
\text { dose of Bu IV. }\end{array}$ & $\begin{array}{l}\text { No patients developed VOD; } \\
\text { acute GVHD developed in } 11 \\
\text { patients (grades } 1-2 \text { in } 10, \\
\text { grade } 11 \text { in } 1 \text { ). }\end{array}$ & $\begin{array}{l}\mathrm{Bu} \text { IV q24 } \mathrm{h} \text { is feasible, safe, and } \\
\text { convenient for administration to children. }\end{array}$ \\
\hline $\begin{array}{l}\text { Adults with metastatic } \\
\text { renal cell carcinoma }\end{array}$ & $\begin{array}{l}\mathrm{Bu} 3.2 \mathrm{mg} / \mathrm{kg} \text { IV } \mathrm{q} 24 \mathrm{~h} \times 2 \text { days }+ \text { Flu } 30 \\
\mathrm{mg} / \mathrm{m}^{2} \mathrm{IV} \times 5 \text { days }(\mathrm{n}=7)\end{array}$ & $\begin{array}{l}\text { AUC was higher than } \\
\text { predicted from extrapolation } \\
\text { of AUC data for the same } \\
\text { total dose of Bu IV q6 h. }\end{array}$ & $\begin{array}{l}\text { Patients experienced } \\
\text { greater than expected } \\
\text { regimen-related toxicity for a } \\
\text { reduced-intensity preparative } \\
\text { regimen, and the study was } \\
\text { stopped. }\end{array}$ & $\begin{array}{l}\text { Bu IV q24 } \mathrm{h} \text { is associated with } \\
\text { unacceptable toxicity compared to Bu IV } \\
\text { q6 } \mathrm{h} \text {, but may be related to fludarabine } \\
\text { exposure and having a single kidney. }\end{array}$ \\
\hline $\begin{array}{l}\text { Pediatrics with } \\
\text { malignant and non- } \\
\text { malignant conditions }\end{array}$ & $\begin{array}{l}\text { 1. Bu } 80 \mathrm{mg} / \mathrm{m}^{2} \text { q2 } 24 \text { h IV+Cy } 200 \mathrm{mg} / \mathrm{kg} \\
\text { IV+Flu } 150 \mathrm{mg} / \mathrm{m}^{2} \text { IV }(\mathrm{n}=7) \\
\text { 2. Bu } 80 \mathrm{mg} / \mathrm{m}^{2} \mathrm{q} 24 \mathrm{~h} \mathrm{IV+Cy} 120 \mathrm{mg} / \mathrm{kg} \\
\text { IV+Mel } 140 \mathrm{mg} / \mathrm{m}^{2} \mathrm{IV}(\mathrm{n}=11)\end{array}$ & $\begin{array}{l}\text { No accumulation occurred } \\
\text { and drug was cleared at } 24 \\
\text { hours. }\end{array}$ & $\begin{array}{l}\text { No new or unexpected } \\
\text { unusual toxicity. }\end{array}$ & $\begin{array}{l}\text { Bu IV q24 } \mathrm{h} \text { in children is safe and } \\
\text { convenient, and can be dosed based on } \\
\text { BSA regardless of age. }\end{array}$ \\
\hline $\begin{array}{l}\text { Adults with AML, MDS, } \\
\text { or CML }\end{array}$ & $\begin{array}{l}\text { 1. Bu } 130 \mathrm{mg} / \mathrm{m}^{2} \mathrm{q} 24 \mathrm{~h} \mathrm{IV} \times 4 \text { days } \\
(\mathrm{n}=60) \\
\text { 2. Bu } 80 \mathrm{mg} / \mathrm{kg} \text { q6 } \mathrm{h} \mathrm{IV} \times 4 \text { days }+\mathrm{Cy} \\
60 \mathrm{mg} / \mathrm{kg} \mathrm{IV} \times 2 \text { days }(\mathrm{n}=47)\end{array}$ & $\begin{array}{l}\text { There was no change } \\
\text { in estimated clearance, } \\
\text { and negligible variability } \\
\text { in dose-to-dose PK or } \\
\text { interdose accumulation. }\end{array}$ & $\begin{array}{l}\text { There was no increase in } \\
\text { any toxicity, particularly } \\
\text { neurotoxicity. }\end{array}$ & $\begin{array}{l}\text { Bu IV has highly predictable, linear PK, } \\
\text { and, Bu q24 h IV is more convenient. }\end{array}$ \\
\hline $\begin{array}{l}\text { Adults with ALL or AML } \\
\text { in } 1^{\text {st }} \text { and } 2^{\text {nd }} \text { remission }\end{array}$ & $\begin{array}{l}\mathrm{Bu} 3.2 \mathrm{mg} / \mathrm{kg} \text { q2 } 24 \mathrm{~h} \mathrm{IV} \times 4 \text { days+Flu } 50 \\
\mathrm{mg} / \mathrm{m}^{2} \mathrm{IV}+\mathrm{TBI} 200 \mathrm{cGy} \times 2 \text { days }\end{array}$ & $\begin{array}{l}\text { PK analysis was not } \\
\text { performed. }\end{array}$ & $\begin{array}{l}\text { The regimen was well- } \\
\text { tolerated. }\end{array}$ & $\begin{array}{l}\text { Bu IV q24 h+Flu +TBI is well tolerated } \\
\text { and gives equivalent final outcomes from } \\
\text { match-related and alternate donors. }\end{array}$ \\
\hline $\begin{array}{l}\text { Adults with AML, ALL, } \\
\text { CML, MDS, or other }\end{array}$ & 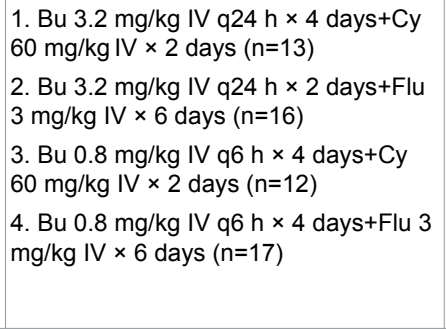 & $\begin{array}{l}\text { PK parameters were not } \\
\text { statistically significantly } \\
\text { different except for } C_{\max } \text {. }\end{array}$ & $\begin{array}{l}\text { There were no significant } \\
\text { differences in acute } \\
\text { GVHD and VOD, although } \\
\text { incidence of acute GVHD } \\
\text { was } 31.0 \% \text { in the Bu IV q6 } \\
\mathrm{h} \text { group vs. } 13.8 \% \text { in the Bu } \\
\text { IV q24 h group ( } \mathrm{p}=0.145 \text { ). } \\
\text { Other toxicities observed } \\
\text { within } 100 \text { days after } \\
\text { transplantation were not } \\
\text { significantly different. }\end{array}$ & $\begin{array}{l}\text { PK profiles and posttransplant } \\
\text { complications are similar for Bu IV q24 } \\
\text { h and Bu IV q6 h with similar number of } \\
\text { posttransplant deaths and overall survival. }\end{array}$ \\
\hline $\begin{array}{l}\text { Pediatrics with } \\
\text { malignant and non- } \\
\text { malignant conditions }\end{array}$ & $\begin{array}{l}\text { 1. Bu } 120 \mathrm{mg} / \mathrm{m}^{2} \mathrm{IV} q 24 \mathrm{~h} \times 4 \text { days }(\mathrm{n}=30) \\
\text { 2. Bu } 1 \mathrm{mg} / \mathrm{kg} \text { PO q6 } \mathrm{h} \times 4 \text { days }(\mathrm{n}=31) \\
\text { 3. All also received either Cy+Mel, Cy, } \\
\text { Flu, or CY+VP16 }\end{array}$ & $\begin{array}{l}\text { The mean total AUC was } \\
\text { higher than the target, } \\
\text { indicating that PK analysis } \\
\text { is necessary for interdose } \\
\text { adjustment. }\end{array}$ & $\begin{array}{l}\text { Bu IV q24 h was associated } \\
\text { with more cases of VOD in } \\
\text { patients who also received } \\
\text { Cy and Mel. }\end{array}$ & $\begin{array}{l}\text { Bu IV q24 h resulted in higher event-free } \\
\text { and overall survival compared to Bu PO. }\end{array}$ \\
\hline Review article & $\begin{array}{l}\text { 1. Bu IV } \\
\text { 2. Bu PO }\end{array}$ & $\begin{array}{l}\text { No accumulation or } \\
\text { increased exposure occurs } \\
\text { with Bu IV q24 h dosing. }\end{array}$ & $\begin{array}{l}\text { Bu IV q24 } \mathrm{h} \text { is associated } \\
\text { with less GI toxicity/ } \\
\text { stomatitis and acute GVHD } \\
\text { compared to q } 6 \text { h dosing. }\end{array}$ & $\begin{array}{l}\text { Bu IV q24 h in myeloablative doses } \\
\text { appear equally effective and perhaps more } \\
\text { convenient compared to Bu IV q6 h. }\end{array}$ \\
\hline $\begin{array}{l}\text { Adults with } \mathrm{AML}, \mathrm{MDS} \\
\mathrm{CML} \text {, Iymphoma, MM, } \\
\text { or other }\end{array}$ & $\begin{array}{l}\text { 1. Bu } 3.2 \mathrm{mg} / \mathrm{kg} \text { IV } q 24 \mathrm{~h} \times 4 \text { days }(\mathrm{n}=22) \\
\text { 2. } \mathrm{Bu} 0.8 \mathrm{mg} / \mathrm{kg} \text { IV q6 } \mathrm{h} \times 4 \text { days }(\mathrm{n}=24)\end{array}$ & $\begin{array}{l}\text { Clearancevolume of } \\
\text { distribution, and half-life did } \\
\text { not differ between the two } \\
\text { groups. }\end{array}$ & $\begin{array}{l}\text { There are no differences } \\
\text { in the incidence of VOD or } \\
\text { elevated bilirubin between } \\
\mathrm{q} 24 \mathrm{~h} \text { and } \mathrm{q} 6 \mathrm{~h} \text { dosing }\end{array}$ & $\begin{array}{l}\text { PK parameters are linear, stable, and } \\
\text { predictable, and unaffected by co- } \\
\text { administration with Flu. }\end{array}$ \\
\hline
\end{tabular}




\begin{tabular}{|c|c|c|c|c|}
\hline $\begin{array}{l}\text { Adults with AML, ALL, } \\
\text { CML, MDS, or other }\end{array}$ & $\begin{array}{l}\text { 1. Bu } 3.2 \mathrm{mg} / \mathrm{kg} \text { IV q24 h } \times 4 \text { days }(n=30) \\
\text { 2. Bu } 0.8 \mathrm{mg} / \mathrm{kg} \text { IV q6 } \mathrm{h} \times 4 \text { days }(\mathrm{n}=30) \\
\text { 3. All also received either Cy, Flu, or Bu } \\
\text { only }\end{array}$ & $\begin{array}{l}\text { PK parameters were used } \\
\text { to develop a new dosing } \\
\text { scheme for Bu IV q24 h. }\end{array}$ & Not assessed. & $\begin{array}{l}\text { Bu dosed at } 24.79^{*} \mathrm{ABW}^{0.5} \mathrm{mg} \text { IV q24 } \\
\mathrm{h} \text { reduces interindividual variability in } \\
\text { clearance and volume of distribution. }\end{array}$ \\
\hline $\begin{array}{l}\text { Adults with AML, ALL, } \\
\text { CML, NHL, MM, HD, } \\
\text { MDS, myelofibrosis, or } \\
\text { other }\end{array}$ & $\begin{array}{l}\text { 1. Bu } 3.2 \mathrm{mg} / \mathrm{kg} \mathrm{IV} \mathrm{q24} \mathrm{h} \times 4 \text { days }+ \text { Flu } \\
50 \mathrm{mg} / \mathrm{m}^{2} \times 5 \text { days }(\mathrm{n}=335) \\
\text { 2. Different test dose strategies } \\
\text { evaluated }\end{array}$ & $\begin{array}{l}\text { Test and treatment dose } \\
\text { should be infused at the } \\
\text { same rate to accurately } \\
\text { predict therapeutic doses. }\end{array}$ & Not assessed. & $\begin{array}{l}\text { A test dose for Bu IV q } 24 \mathrm{~h} \text { is accurate as } \\
\text { long as it is infused at the same rate as the } \\
\text { treatment dose. }\end{array}$ \\
\hline Adults with ALL & $\begin{array}{l}\mathrm{Bu} 3.2 \mathrm{mg} / \mathrm{kg} \text { IV q24 } \mathrm{h} \times 4 \text { days }+ \text { Clo } 40 \\
\mathrm{mg} / \mathrm{m}^{2} \mathrm{IV} \times 1 \text { day }(\mathrm{n}=51)\end{array}$ & $\begin{array}{l}\text { PK-guided Bu IV led to } \\
\text { accurate dose delivery } \\
\text { within a tight therapeutic } \\
\text { window. }\end{array}$ & $\begin{array}{l}\text { Bu IV q24 h in combination } \\
\text { with Clo is well-tolerated with } \\
\text { no cases of VOD. }\end{array}$ & $\begin{array}{l}\text { Bu IV q24 } \mathrm{h}+\mathrm{Clo} \text { produces excellent } \\
\text { disease control in } \mathrm{ALL} \text { with favorable } \\
\text { toxicity profile. }\end{array}$ \\
\hline $\begin{array}{l}\text { Adults with } A M L \\
\text { MDS, CML, CLL, } \\
\text { myelofibrosis, or other }\end{array}$ & $\begin{array}{l}\text { 1. Bu } 3.2 \mathrm{mg} / \mathrm{kg} \text { IV q24 h } \times 4 \text { days }+ \text { Cy } \\
60 \mathrm{mg} / \mathrm{kg} \text { IV } \times 2 \text { days }(\mathrm{n}=42) \\
\text { 2. Bu } 3.2 \mathrm{mg} / \mathrm{kg} \text { IV q24 } \mathrm{h} \times 4 \text { days }+F l u \\
30 \mathrm{mg} / \mathrm{m}^{2} \mathrm{IV} \times 4 \text { days }+\mathrm{rATG}(\mathrm{n}=29) \\
3 . \mathrm{Bu} 3.2 \mathrm{mg} / \mathrm{kg} \text { IV q24 } \mathrm{h} \times 4 \text { days+Flu } \\
50 \mathrm{mg} / \mathrm{m}^{2} \mathrm{IV} \times 5 \text { days }+\mathrm{rATG}(\mathrm{n}=16)\end{array}$ & $\begin{array}{l}\text { Bu } 3.2 \mathrm{mg} / \mathrm{kg} \text { IV q24 h led to } \\
\text { suboptimal } \mathrm{C}_{\mathrm{sS}} \text {, while Bu } 4 \\
\mathrm{mg} / \mathrm{kg} \text { achieved the desired } \\
\mathrm{C}_{\mathrm{ss}} \text {. There is minimal dose- } \\
\text { to-dose variability of busulfan } \\
\text { clearance. }\end{array}$ & Not assessed. & $\begin{array}{l}\text { TDM should be conducted by sampling } \\
\text { over a minimum of } 8 \text { hours after the start } \\
\text { of infusion. }\end{array}$ \\
\hline $\begin{array}{l}\text { Adults with } \mathrm{CML}, \mathrm{CLL} \\
\text { MDS, MM, NHL, HD, } \\
\text { myelofibrosis, or other }\end{array}$ & $\begin{array}{l}\mathrm{Bu} 3.2 \mathrm{mg} / \mathrm{kg} \text { IV q24 } \mathrm{h} \times 2 \text { days }+ \text { Flu } 50 \\
\mathrm{mg} / \mathrm{m}^{2} \mathrm{IV} \times 5 \text { days }(\mathrm{n}=158)\end{array}$ & $\begin{array}{l}\text { Target AUC was achieved } \\
\text { and led to improved } \\
\text { outcomes when daily Bu } \\
\text { exposure was } 3814-4994 \\
\mu \mathrm{M} / \mathrm{min} \text {. }\end{array}$ & $\begin{array}{l}\text { Patients achieving target } \\
\text { AUC had the lowest rates of } \\
\text { acute GVHD. }\end{array}$ & $\begin{array}{l}\text { Bu IV q24 } \mathrm{h} \text { is effective in achieving } \\
\text { targeted AUC and yields similar outcomes } \\
\text { to q6 } \mathrm{h} \text { dosing. Target AUC may differ } \\
\text { based on the conditioning protocol and the } \\
\text { disease. }\end{array}$ \\
\hline $\begin{array}{l}\text { Adults with relapsed } \\
\text { MM }\end{array}$ & $\begin{array}{l}\text { Bu } 3.2 \mathrm{mg} / \mathrm{kg} \text { IV q24 } \mathrm{h} \times 2 \text { days+Btz } \\
1.3 \mathrm{mg} / \mathrm{m}^{2} \times 1 \text { day }(\mathrm{n}=30)\end{array}$ & $\begin{array}{l}\text { An outpatient test dose of } \\
\text { Bu IV led to only } 2 \text { of } 30 \\
\text { patients requiring dose } \\
\text { adjustment based on } \\
\text { confirmatory PK. }\end{array}$ & $\begin{array}{l}\text { No abnormal toxicity was } \\
\text { noted among patients } \\
\text { achieving target AUC. }\end{array}$ & $\begin{array}{l}\text { Targeted dose Bu IV q24 } \mathrm{h} \text { in combination } \\
\text { with bortezomib is effective with } \\
\text { acceptable toxicity. }\end{array}$ \\
\hline $\begin{array}{l}\text { Adults with ALL, AML, } \\
\text { CML, NHL, or other }\end{array}$ & $\begin{array}{l}\text { 1. Bu } 130 \mathrm{mg} / \mathrm{m}^{2} \mathrm{IV} \mathrm{q} 24 \mathrm{~h} \times 4 \text { days }+\mathrm{Flu} \\
40 \mathrm{mg} / \mathrm{m}^{2} \mathrm{IV} \times 4 \text { days }(\mathrm{n}=18) \\
\text { 2. Bu } 0.8 \mathrm{mg} / \mathrm{kg} \text { IV } \mathrm{q} 6 \mathrm{~h} \times 4 \text { days }+\mathrm{Cy} \\
60 \mathrm{mg} / \mathrm{kg} \mathrm{IV} \times 2 \text { days }(\mathrm{n}=13)\end{array}$ & $\begin{array}{l}\text { All patients required dose } \\
\text { adjustment to achieve } \\
\text { target AUC, but using } \\
\text { actual body weight led to } \\
\text { more accurate dosing. }\end{array}$ & $\begin{array}{l}\text { No patients experienced } \\
\text { busulfan-related toxicity. }\end{array}$ & $\begin{array}{l}\text { No differences were noted related to } \\
\text { dosing frequency; further study needed to } \\
\text { determine optimal weight to achieve target } \\
\text { AUC. }\end{array}$ \\
\hline $\begin{array}{l}\text { Adults with AML, } \\
\text { ALL, MDS, Hodgkin's } \\
\text { lymphoma, or other }\end{array}$ & $\begin{array}{l}\text { 1. Bu } 3.2 \mathrm{mg} / \mathrm{kg} \text { IV q } 24 \mathrm{~h} \times 4 \text { days }+ \text { Cy } \\
60 \mathrm{mg} / \mathrm{kg} \mathrm{IV} \times 2 \text { days } \\
\text { 2. Bu } 3.2 \mathrm{mg} / \mathrm{kg} \text { IV q } 24 \mathrm{~h} \times 4 \text { days+Flu } \\
30 \mathrm{mg} / \mathrm{m}^{2} \mathrm{IV} \times 6 \text { days } \\
3 . \mathrm{Bu} 3.2 \mathrm{mg} / \mathrm{kg} \text { IV q } 24 \mathrm{~h} \times 4 \text { days+Mel } \\
140 \mathrm{mg} / \mathrm{m}^{2} \times 1 \text { day }\end{array}$ & $\begin{array}{l}\text { Median AUC was similar } \\
\text { to other studies of Bu IV } \\
\text { q24 h and estimated daily } \\
\text { AUC based on Bu IV q6 h } \\
\text { studies. }\end{array}$ & $\begin{array}{l}\text { No patients experienced } \\
\text { VOD, and no regimen- } \\
\text { related toxicity was } \\
\text { significantly associated with } \\
\text { AUC. }\end{array}$ & $\begin{array}{l}\text { Bu IV q24 } \mathrm{h} \text { has a stable PK profile and is } \\
\text { more convenient than } \mathrm{q} 6 \mathrm{~h} \text { dosing. }\end{array}$ \\
\hline Adults eligible for HSCT & $\begin{array}{l}\text { 1. Bu } 3.2 \mathrm{mg} / \mathrm{kg} \text { IV q24 } \mathrm{h} \times 2 \text { days+Flu } \\
30 \mathrm{mg} / \mathrm{m}^{2} \mathrm{IV} \times 5 \text { days }(\mathrm{n}=19) \\
\text { 2. Bu }\left(23 \times \mathrm{AB}^{0.5}\right) \mathrm{mg} \mathrm{IV} \mathrm{q} 24 \mathrm{~h}(\mathrm{n}=18) \\
\text { 3. All also received either Flu+ATG, } \\
\text { Cy, or Flu }\end{array}$ & $\begin{array}{l}\text { Using a population PK } \\
\text { model with dosage history } \\
\text { and demographic data can } \\
\text { optimize the busulfan dose } \\
\text { required to achieve target } \\
\text { AUC. }\end{array}$ & Not assessed. & $\begin{array}{l}\text { Bu IV q24 h dosed using a novel scheme } \\
\text { based on actual body weight may result in } \\
\text { better PK compared to } 3.2 \mathrm{mg} / \mathrm{kg} \text { dosing. }\end{array}$ \\
\hline $\begin{array}{l}\text { Adults with NHL or } \\
\text { Hodgkins lymphoma }\end{array}$ & $\begin{array}{l}\text { 1. } B u 2.8 \mathrm{mg} / \mathrm{kg} \text { IV q24 } \mathrm{h} \times 4 \\
\text { days }+C y+V P 16 \text { IV } \times 5 \text { days }(\mathrm{n}=93) \\
\text { 2. Bu } 0.8 \mathrm{mg} / \mathrm{kg} \text { IV q6 } \mathrm{h} \times 3.5 \text { days } \\
(\mathrm{n}=307)\end{array}$ & $\begin{array}{l}\text { There is a significant range } \\
\text { of AUCs with a standard } \\
\text { deviation of } 13 \% \text {. }\end{array}$ & $\begin{array}{l}\text { There was no increase in } \\
\text { pulmonary or hepatic toxicity. }\end{array}$ & $\begin{array}{l}\text { Bu IV q24 } \mathrm{h} \text { is more convenient than Bu IV } \\
\text { q6 } \mathrm{h} \text { with equivalent clinical outcomes. }\end{array}$ \\
\hline $\begin{array}{l}\text { Practice Guidelines } \\
\text { Committee of the } \\
\text { American Society } \\
\text { of Blood or Marrow } \\
\text { Transplantation (ASBMT) } \\
\end{array}$ & $\begin{array}{l}\text { N/A; the document is a compilation of } \\
\text { FAQ's regarding personalized busulfan } \\
\text { dosing }\end{array}$ & $\begin{array}{l}\text { Bu clearance, volume of } \\
\text { distribution, and half-life } \\
\text { are similar regardless of } \\
\text { whether Bu IV is given q } 6 \mathrm{~h} \\
\text { or q24 } \mathrm{h} \text {. }\end{array}$ & $\begin{array}{l}\text { It has been demonstrated } \\
\text { that } \mathrm{q} 24 \mathrm{~h} \text { dosing is safe. }\end{array}$ & $\begin{array}{l}\text { Bu IV q24 } \mathrm{h} \text { is clinically equivalent to Bu } \\
\text { IV q6 } \mathrm{h} \text {. }\end{array}$ \\
\hline $\begin{array}{l}\text { Adults with AML, CML } \\
\text { or MDS }\end{array}$ & $\begin{array}{l}\text { 1. Bu IV q6 } h \times 4 \text { days }+ \text { Cy }(n=495)^{*} \\
\text { 2. Bu IV q24 } \mathrm{h} \times 4 \text { days+Flu }(n=331)^{*} \\
\text { 3. Bu IV q24 h+Cy }(n=96)^{*} \\
\text { 4. Bu IV q6h+Flu }(n=91)^{*}\end{array}$ & $\begin{array}{l}\text { There are no differences } \\
\text { between Bu IV q24 h and } \\
\text { Bu IV q6 h. }\end{array}$ & $\begin{array}{l}\text { Cumulative incidence of } \\
\text { VOD, idiopathic pneumonia, } \\
\text { and seizures did not differ } \\
\text { across groups. }\end{array}$ & $\begin{array}{l}\text { Bu IV q24 } \mathrm{h} \text { and Bu IV q6 } \mathrm{h} \text { alone or in } \\
\text { combination with Cy or Flu have similar } \\
\text { outcomes in the myeloablative setting for } \\
\text { treatment of myeloid malignancies. }\end{array}$ \\
\hline
\end{tabular}

ALL: Acute Lymphocytic Leukemia; AML: Acute Myelogenous Leukemia; AUC: Area Under the Curve; CLL: Chronic Lymphocytic Leukemia; CML: Chronic Myelogenous Leukemia; GVHD: Graft-Versus-Host Disease; HD: Hodgkin's Disease; HSCT: Hematopoietic Stem Cell Transplant; MDS: Myelodysplastic Syndrome; MM: Multiple Myeloma; NHL: Non-Hodgkin's Lymphoma; VOD: Veno-Occlusive Disease; TDM: Therapeutic Dose Monitoring.

*Dosing unspecified

Table 1: A summary of relevant literature.

hepatotoxicity, engraftment, and overall survival [37]. However, there are still significant and common adverse effects that may affect the patient's quality of life that may not be reflected in those categories. These are important to look into further as we continue to adopt this approach. Although existing literature describes major toxicities associated with busulfan (i.e., seizures and sinusoidal obstructive syndrome (SOS) that are equivalent when pharmacokinetic monitoring assumes equal AUC concentrations, a larger study is warranted to verify these claims $[5,40,41]$.

Busulfan is available in $60 \mathrm{mg}$ vials, and is typically dosed at a starting dose of $0.8 \mathrm{mg} / \mathrm{kg} \mathrm{q} 6 \mathrm{~h}$, or $3.2 \mathrm{mg} / \mathrm{kg} \mathrm{q} 24 \mathrm{~h}$ (with subsequent 
pharmacokinetic adjustment) for a total of four days as part of a myeloablative regimen prior to HCT [4]. With q6 h dosing, a typical $75 \mathrm{~kg}$ patient would receive $67.5 \mathrm{mg}$ per dose, therefore requiring 2 vials per dose and 8 vials per day, for a total of 32 vials per patient per treatment course. Using less frequent $\mathrm{q} 24 \mathrm{~h}$ dosing, the same patient would receive a typical dose of $270 \mathrm{mg}$ per day, requiring 5 vials per day for a total of 20 vials per patient per treatment course. Reducing the dosing frequency to daily administration therefore results in a $63 \%$ reduction in the necessary busulfan purchasing in this typical situation (from 1,920 mg to $1,200 \mathrm{mg}$ ), translating into significant direct drug cost savings with identical clinical efficacy. Busulfan is a relatively expensive medication; according to the Veterans Affairs Federal Supply Schedule, the intravenous form costs approximately $\$ 935.72$ per vial in the US, implying that converting from $\mathrm{q} 6 \mathrm{~h}$ to $\mathrm{q} 24 \mathrm{~h}$ dosing could save at least $\$ 11,000$ per patient in direct drug costs.

Not only does the reduced dosing frequency yield direct drug cost savings, but it also decreases pharmacy resources required to prepare the IV admixture from four times daily, to just once per day. In particular, busulfan's stability is only 12 hours once admixed, so daily administration is more practical for pharmacy [34]. Nursing administration is also simplified with a once-daily regimen. Nursing and pharmacist workloads have been shown to impact medication safety, provider burnout, and job satisfaction [42,43]. Switching from $\mathrm{q} 6 \mathrm{~h}$ to $\mathrm{q} 24 \mathrm{~h}$ busulfan administration can thus have a positive impact on both patient safety and provider satisfaction. Finally, as patient satisfaction is increasingly utilized as an important metric of care quality, it seems reasonable to assume (although it remains to be proven) that patient satisfaction will increase with fewer interruptions and less frequent medication administration.

In an informal poll of 24 institutions that perform HCT, we found that $20(83.3 \%)$ were already utilizing $\mathrm{q} 24 \mathrm{~h}$ dosing. Although this was an informal poll with a small sample size, the results imply that institutions are moving towards this practice, but also that $\mathrm{q} 6 \mathrm{~h}$ dosing remains in use at a significant minority of large transplant centers.

\section{Conclusion}

Based on the available literature, busulfan IV q24 h is comparable to $\mathrm{q} 6 \mathrm{~h}$ dosing in both safety and efficacy. Future research should focus on busulfan dosing in pediatric patients, given that the current evidence for once-daily busulfan is not as compelling as that for adults. Another important area for research should focus on identifying the significant differential toxicities associated with $\mathrm{q} 6 \mathrm{~h}$ vs. $\mathrm{q} 24 \mathrm{~h}$ dosing. Institutions utilizing busulfan $\mathrm{q} 6 \mathrm{~h}$ dosing should consider switching their practice to $\mathrm{q} 24 \mathrm{~h}$ to reduce costs and administration resources, and improve patient quality of life.

\section{References}

1. US Food and Drug Administration (1954) Myleran New Drug Application (NDA) 009386. FDA Approved Drug Products.

2. Tutschka PJ, Copelan EA, Klein JP (1987) Bone marrow transplantation for leukemia following a new busulfan and cyclophosphamide regimen. Blood 70: 1382-1388.

3. Thomas ED, Clift RA, Fefer A, Appelbaum FR, Beatty P, et al. (1986) Marrow transplantation for the treatment of chronic myelogenous leukemia. Annals of Internal Medicine 104: 155-163.

4. Palmer J, McCune JS, Perales MA, Marks D, Bubalo J, et al. (2016) Personalizing busulfan-based conditioning: considerations from the American society for blood and marrow transplantation practice guidelines committee. Biology of Blood and Marrow Transplantation 22: 1915-1925.

5. Pasquini MC, Le-Rademacher J, Zhu X, Artz A, DiPersio J, et al. (2016)
Intravenous busulfan-based myeloablative conditioning regimens prior to hematopoietic cell transplantation for hematologic malignancies. Biology of Blood and Marrow Transplantation 22: 1424-1430.

6. Glaxo Smith Kline LLC (2011) Myleran: Package Insert and Label Information. Feucht Germany.

7. Haddow A, Timmis GM (1953) Myleran in chronic myeloid leukaemia Chemical constitution and biological action. The Lancet 261: 207-208.

8. Galton DAG (1953) Myleran in Chronic Myeloid Leukæmia Results of Treatment. The Lancet 261: 208-213.

9. Haut A, Altman SJ, Cartwright GE, Wintrobe MM (1955) The use of myleran in the treatment of chronic myelocytic leukemia. AMA Archives of Internal Medicine 96: 451-462.

10. Balasubramanian P, Desire S, Panetta JC, Lakshmi KM, Mathews V, et al. (2012) Population pharmacokinetics of cyclophosphamide in patients with thalassemia major undergoing HSCT. Bone Marrow Transplantation 47: 1178

11. Chandy M, Balasubramanian P, Ramachandran SV, Mathews V, George B et al. (2005) Randomized trial of two different conditioning regimens for bone marrow transplantation in thalassemia-the role of busulfan pharmacokinetics in determining outcome. Bone Marrow Transplantation 36: 839.

12. Hassan M, Öberg G, Bekassy AN, Aschan J, Ehrsson H, et al. (1991) Pharmacokinetics of high-dose busulphan in relation to age and chronopharmacology. Cancer Chemotherapy and Pharmacology 28: 130-134.

13. 13. Takama $H$, Tanaka $H$, Nakashima D, Ueda R, Takaue $Y$ (2006)Population pharmacokinetics of intravenous busulfan in patients undergoing hematopoietic stem cell transplantation. Bone Marrow Transplantation 37: 345.

14. US Food and Drug Administration (1999) Busulfex New Drug Application (NDA): 020954.

15. Bendamustine. Micromedex. Truven Health Analytics; 2018. Accessed January 30, 2018.

16. Busulfan. Micromedex. Truven Health Analytics; 2018. Accessed January 30 , 2018.

17. Carmustine. Micromedex. Truven Health Analytics; 2018. Accessed January 30, 2018.

18. Carboplatin. Micromedex. Truven Health Analytics; 2018. Accessed January 30, 2018.

19. Chlorambucil. Micromedex. Truven Health Analytics; 2018. Accessed January 30, 2018.

20. Cisplatin. Micromedex. Truven Health Analytics; 2018. Accessed January 30, 2018.

21. Cyclophosphamide. Micromedex. Truven Health Analytics; 2018. Accessed January 30,2018

22. Ifosfamide. Micromedex. Truven Health Analytics; 2018. Accessed January 30 2018

23. Melphalan. Micromedex. Truven Health Analytics; 2018. Accessed January 30 2018.

24. Oxaliplatin. Micromedex. Truven Health Analytics; 2018. Accessed January 30 , 2018.

25. Procarbazine. Micromedex. Truven Health Analytics; 2018. Accessed January 30, 2018.

26. 26. Pidala J, Kim J, Anasetti C, Kharfan-Dabaja MA, Nishihori T, et al. (2010) Pharmacokinetic targeting of intravenous busulfan reduces conditioning regimen related toxicity following allogeneic hematopoietic cell transplantation for acute myelogenous leukemia. Journal of Hematology \& Oncology 3: 36.

27. Zhang H, Graiser M, Hutcherson DA, Dada MO, McMillan S, et al. (2012) Pharmacokinetic-directed high-dose busulfan combined with cyclophosphamide and etoposide results in predictable drug levels and durable long-term survival in lymphoma patients undergoing autologous stem cell transplantation. Biology of Blood and Marrow Transplantation 18: 1287-1294.

28. Fasola G, Aprile G, Marini L, Follador A, Mansutti M, et al. (2014) Drug waste minimization as an effective strategy of cost-containment in Oncology. BMC health Services Research 14: 57

29. Xu QA, Zhang YP, Trissel LA, Martinez JF (1996) Stability of busulfan injection 
admixtures in $5 \%$ dextrose injection and $0.9 \%$ sodium chloride injection. Journal of Oncology Pharmacy Practice 2: 101-105.

30. Andersson BS, de Lima M, Thall PF, Wang X, Couriel D, et al. (2008) Once daily iv busulfan and fludarabine (iv Bu-Flu) compares favorably with iv busulfan and cyclophosphamide (iv BuCy2) as pretransplant conditioning therapy in AML/ MDS. Biology of Blood and Marrow Transplantation 14: 672-684.

31. Bartelink IH, Bredius RG, Ververs TT, Raphael MF, van Kesteren C, et al. (2008) Once-daily intravenous busulfan with therapeutic drug monitoring compared to conventional oral busulfan improves survival and engraftment in children undergoing allogeneic stem cell transplantation. Biology of Blood and Marrow Transplantation 14: 88-98.

32. Shaughnessy P, Alexander W, Tran H, Ririe D, Splichal J, et al. (2006) Phase and pharmacokinetic study of once-daily dosing of intravenously administered busulfan in the setting of a reduced-intensity preparative regimen and allogeneic hematopoietic stem cell transplantation as immunotherapy for renal cell carcinoma. Military Medicine 171: 161-165.

33. Kletzel M, Jacobsohn D, Duerst R (2006) Pharmacokinetics of a test dose of intravenous busulfan guide dose modifications to achieve an optimal area under the curve of a single daily dose of intravenous busulfan in children undergoing a reduced-intensity conditioning regimen with hematopoietic stem cell transplantation. Biology of Blood and Marrow Transplantation 12: 472-479.

34. Zwaveling J, den Hartigh J, Lankester AC, Guchelaar HJ, Egeler RM, et al. (2006) Once-daily intravenous busulfan in children prior to stem cell transplantation: study of pharmacokinetics and early clinical outcomes. Anticancer Drugs 17: 1099-1105.

35. Clemmons AB, Evans S, DeRemer DL, Awan FT (2015) Busulfan dosing (Q6 or Q24) with adjusted or actual body weight, does it matter?. Journal of Oncology Pharmacy Practice 21: 425-432.

36. De Lima M, Couriel D, Thall PF, Wang X, Madden T, et al. (2004) Once-daily intravenous busulfan and fludarabine: clinical and pharmacokinetic results of a myeloablative, reduced-toxicity conditioning regimen for allogeneic stem cell transplantation in AML and MDS. Blood 104: 857-864.

37. Hill BT, Rybicki L, Carlstrom KD, Jagadeesh D, Gerds A, et al. (2016) Daily Weight-Based Busulfan with Cyclophosphamide and Etoposide Produces Comparable Outcomes to Four-Times-Daily Busulfan Dosing for Lymphoma Patients Undergoing Autologous Stem Cell Transplantation. Biology of Blood and Marrow Transplantation 22: 1588-1595.

38. 38. Madden T, de Lima M, Thapar N, Nguyen J, Roberson S, et al. (2007) Pharmacokinetics of once-daily IV busulfan as part of pretransplantation preparative regimens: a comparison with an every 6-hour dosing schedule. Biology of Blood and Marrow Transplantation 13: 56-64.

39. Hassan Z, Hassan M, Hellström-Lindberg E (2001) The pharmacodynamic effect of busulfan in the P39 myeloid cell line in vitro. Leukemia 15: 1240.

40. Almog S, Kurnik D, Shimoni A, Loebstein R, Hassoun E, et al. (2011) Linearity and stability of intravenous busulfan pharmacokinetics and the role of glutathione in busulfan elimination. Biology of Blood and Marrow Transplantation 17: 117-123.

41. Ryu SG, Lee JH, Choi SJ, Lee JH, Lee YS, et al. (2007) Randomized comparison of four-times-daily versus once-daily intravenous busulfan in conditioning therapy for hematopoietic cell transplantation. Biology of Blood and Marrow Transplantation 13: 1095-1105.

42. Holden RJ, Scanlon MC, Patel NR, Kaushal R, Escoto KH, et al. (2011) A human factors framework and study of the effect of nursing workload on patien safety and employee quality of working life. BMJ Quality \& Safety 20: 15-24.

43. Holden RJ, Patel NR, Scanlon MC, Shalaby TM, Arnold JM, et al. (2010) Effects of mental demands during dispensing on perceived medication safety and employee well-being: A study of workload in pediatric hospital pharmacies Research in Social and Administrative Pharmacy 6: 293-306. 